\title{
Le GIS « Patrimoines en partage »
}

\section{Sylvie Sagnes}

\section{(2) OpenEdition}

\section{Journals}

Édition électronique

URL : https://journals.openedition.org/insituarss/1423

DOI : 10.4000/insituarss. 1423

ISSN : 2680-4972

\section{Éditeur}

Ministère de la Culture

\section{Référence électronique}

Sylvie Sagnes, «Le GIS « Patrimoines en partage » », In Situ. Au regard des sciences sociales [En ligne], 2 | 2021, mis en ligne le 18 mars 2021, consulté le 23 janvier 2023. URL : http://

journals.openedition.org/insituarss/1423; DOI : https://doi.org/10.4000/insituarss. 1423

Ce document a été généré automatiquement le 23 janvier 2023.

\section{(c) (i) (9)}

Creative Commons - Attribution - Pas d'Utilisation Commerciale - Pas de Modification 4.0 International - CC BY-NC-ND 4.0

https://creativecommons.org/licenses/by-nc-nd/4.0/ 


\title{
Le GIS « Patrimoines en partage »
}

\author{
Sylvie Sagnes
}

1 Longtemps, l'anthropologie occupée de patrimoine est restée aveugle aux opérations de médiation, abandonnant leur étude à d'autres disciplines, au premier rang desquelles les SIC (sciences de l'information et de la communication). Or à y regarder de près, la médiation s'impose comme un lieu et un moment clé : elle illustre, outre le passage de l'«âge du monument " à l'« âge du patrimoine » mis en évidence par les analyses de Daniel Fabre (2016), la coexistence de ces deux âges, au cœur de la modernité patrimoniale, ainsi que les tensions, contradictions qui se font jour entre les deux dispositifs (au sens foucaldien du terme). Car la médiation est l'héritière d'une longue histoire, marquée d'abord par les mots d'ordre que sont éducation populaire et démocratisation culturelle, puis, à compter des années 1960, par les revendications visant à faire droit à la contre-culture et à la démocratie culturelle. Telle que conceptualisée dans les années 1990, à savoir comme un " acte de partage », suivant un mouvement "vers / de l'autre » (Caillet 1994), la médiation entend justement articuler ces deux manières divergentes de concevoir l'action culturelle, et orchestre de fait quelque chose de la coexistence des dispositifs "monument» et "patrimoine». Mais plus qu'un observatoire, les médiations forment un laboratoire de la patrimonialité, en ce sens que les représentations du patrimoine qu'elles mobilisent se trouvent agies dans leur confrontation, c'est-à-dire retenues ou exclues, mêlées, travaillées, remodelées...

2 Le GIS (Groupement d'intérêt scientifique) "Patrimoines en partage » entend privilégier l'observation et l'analyse, au cœur du processus d'institution patrimoniale, de ce moment singulier, qui succède à la mise à distance physique et symbolique des biens culturels et précède l'étape finale de la jouissance individuelle et collective. Abordant le terme non pas dans l'acception stricte que peuvent lui donner, dans le sillage des SIC, les établissements culturels, les milieux de la formation professionnelle ou encore les cadres d'emploi de la fonction publique territoriale, le projet scientifique du GIS conçoit la médiation de manière générique, afin de n'exclure aucune des situations où le patrimoine est donné en partage. Il se propose d'embrasser le vaste horizon d'investigation défini par cette ambition, pour ouvrir dans le champ des heritage studies des voies encore inexplorées. 
Figure 1

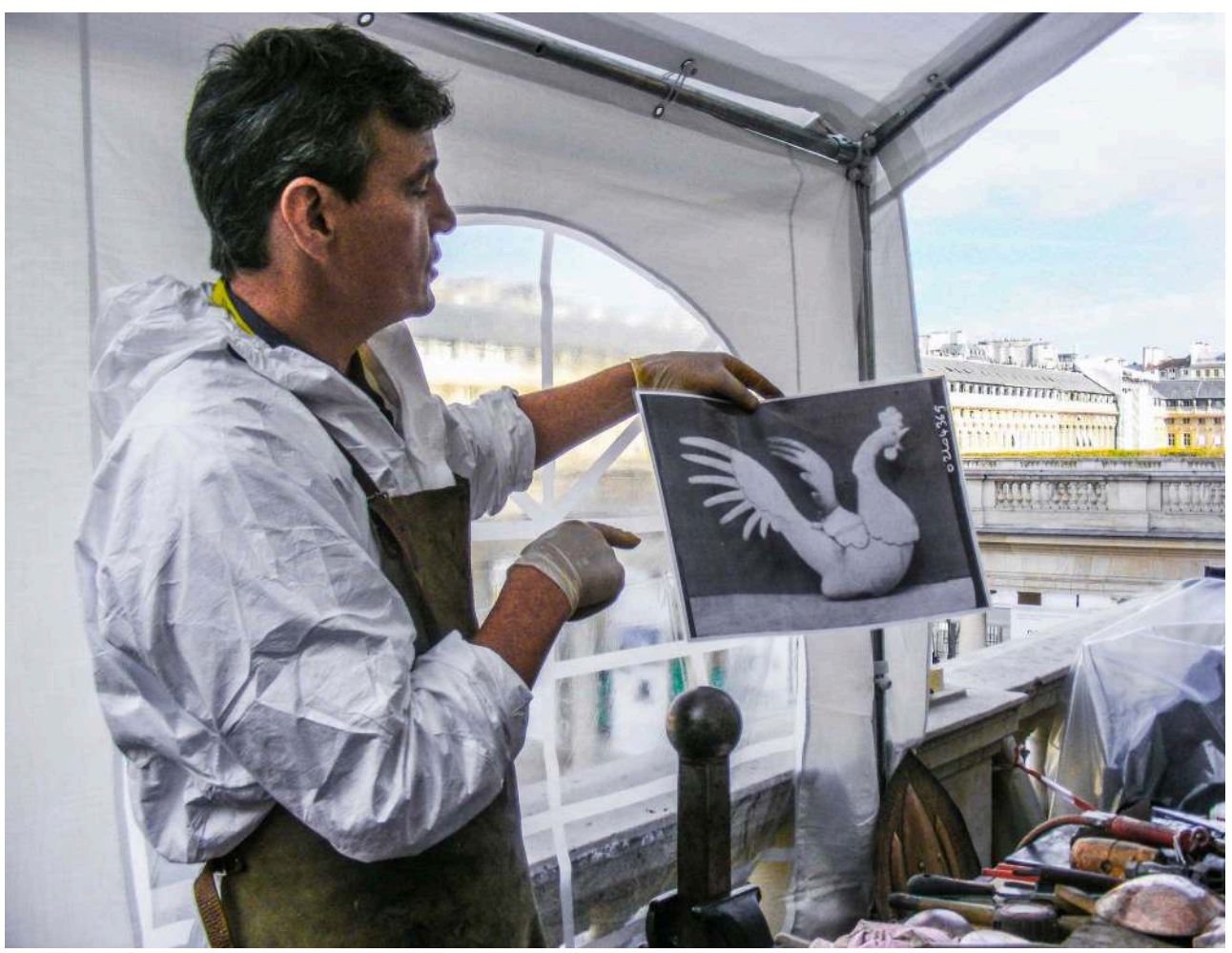

Démonstration par la Socra des savoir-faire relatifs à la statuaire de métal (exposition « Revoir NotreDame », Journées européennes du patrimoine, 2019), l'une des médiations de la cathédrale en absence, thématique du chantier-école Notre-Dame de Paris, rattaché au GIS « Patrimoines en partage ».

(C) Sylvie Sagnes. 
Figure 2

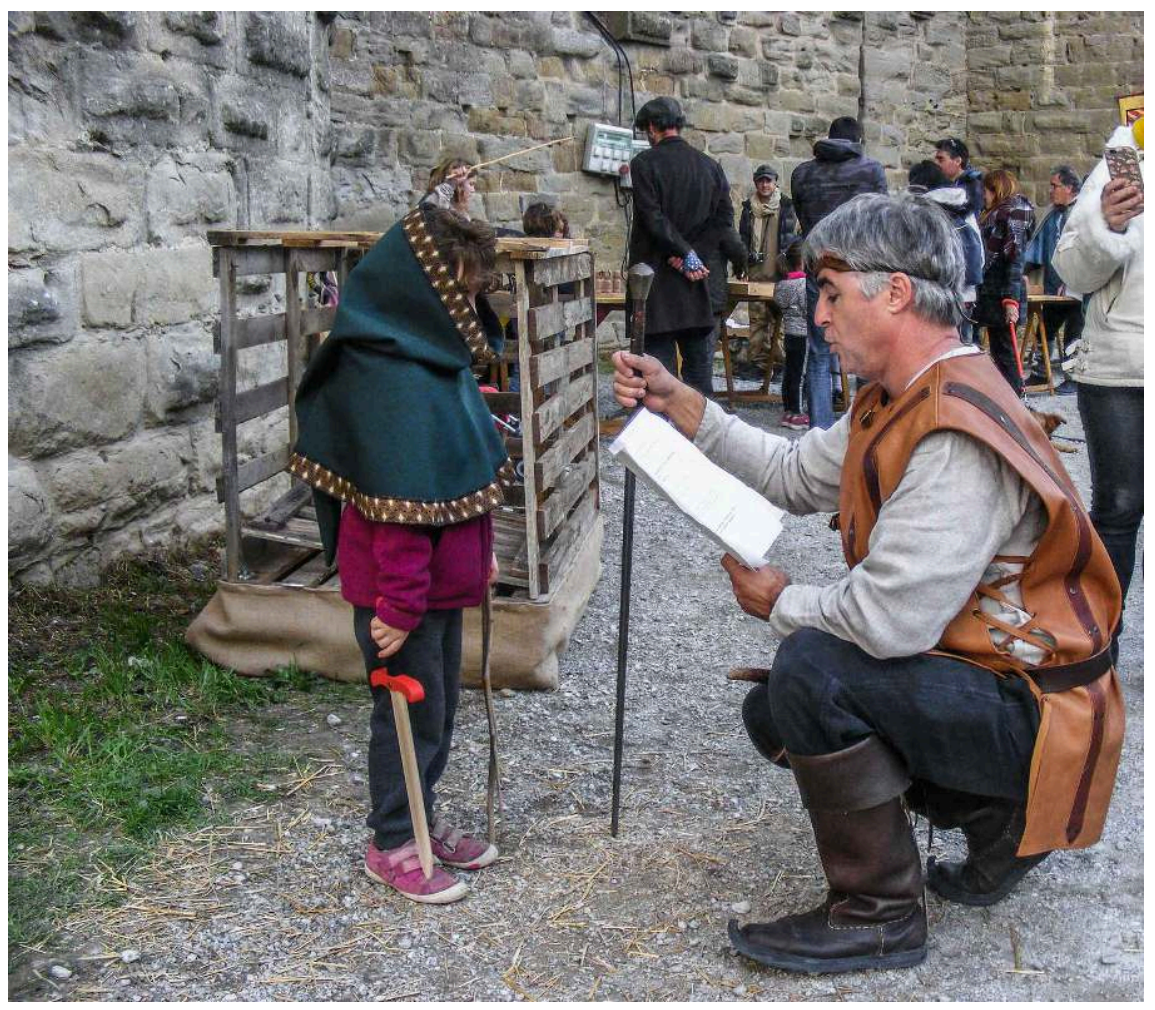

Scène d'adoubement au cours du Téléthon 2019 à la Cité de Carcassonne, ou comment faire vivre le Moyen Âge ? Tel fut le thème du colloque de préfiguration 2019 du GIS « Patrimoines en partage » : «Vivre et faire vivre le Moyen Âge » (LERASS / Ethnopôle Garae).

(c) Sylvie Sagnes.

3 L'idée de ce GIS a germé en 2017 dans un terreau un peu inhabituel pour ce type de projet, à savoir au cœur d'un ethnopôle, en l'occurrence le Garae (Groupe audois de recherche et d'animation ethnographique). Créée en 1981 à Carcassonne et labellisée Ethnopôle en 1996 par le ministère de la Culture, cette association loi 1901 a été très tôt partie-prenante du retournement conduisant l'anthropologie à envisager le patrimoine autrement, à savoir comme un trait culturel majeur de notre modernité, de sorte qu'au cours des deux dernières décennies, l'« ethnologie du patrimoine » a formé la colonne vertébrale de ses activités de recherche et de sa politique partenariale. Celles-ci le lient de manière très étroite au Lahic (Laboratoire d'anthropologie et d'histoire sur l'institution de la culture, intégré à en 2006 l'IIAC-Institut interdisciplinaire d'anthropologie du contemporain), ce qui n'exclut pas d'autres collaborations, notamment avec le Centre d'anthropologie (Toulouse), le Cerce (Centre d'études et de recherches comparatives en ethnologie, Montpellier) et l'Idemec (Institut d'ethnologie méditerranéenne, européenne et comparative, Aix-Marseille). Mais le Garae ne saurait être réductible aux travaux de recherche qu'il suscite ou accueille, dans la mesure où se trouve aussi inscrit dans son ADN un réel souci d'implication dans le panorama du patrimoine en acte, qui hisse au premier rang de ses préoccupations la médiation de la recherche en ethnologie en général et de la notion de patrimoine culturel immatériel (et de ses expressions) en particulier. 


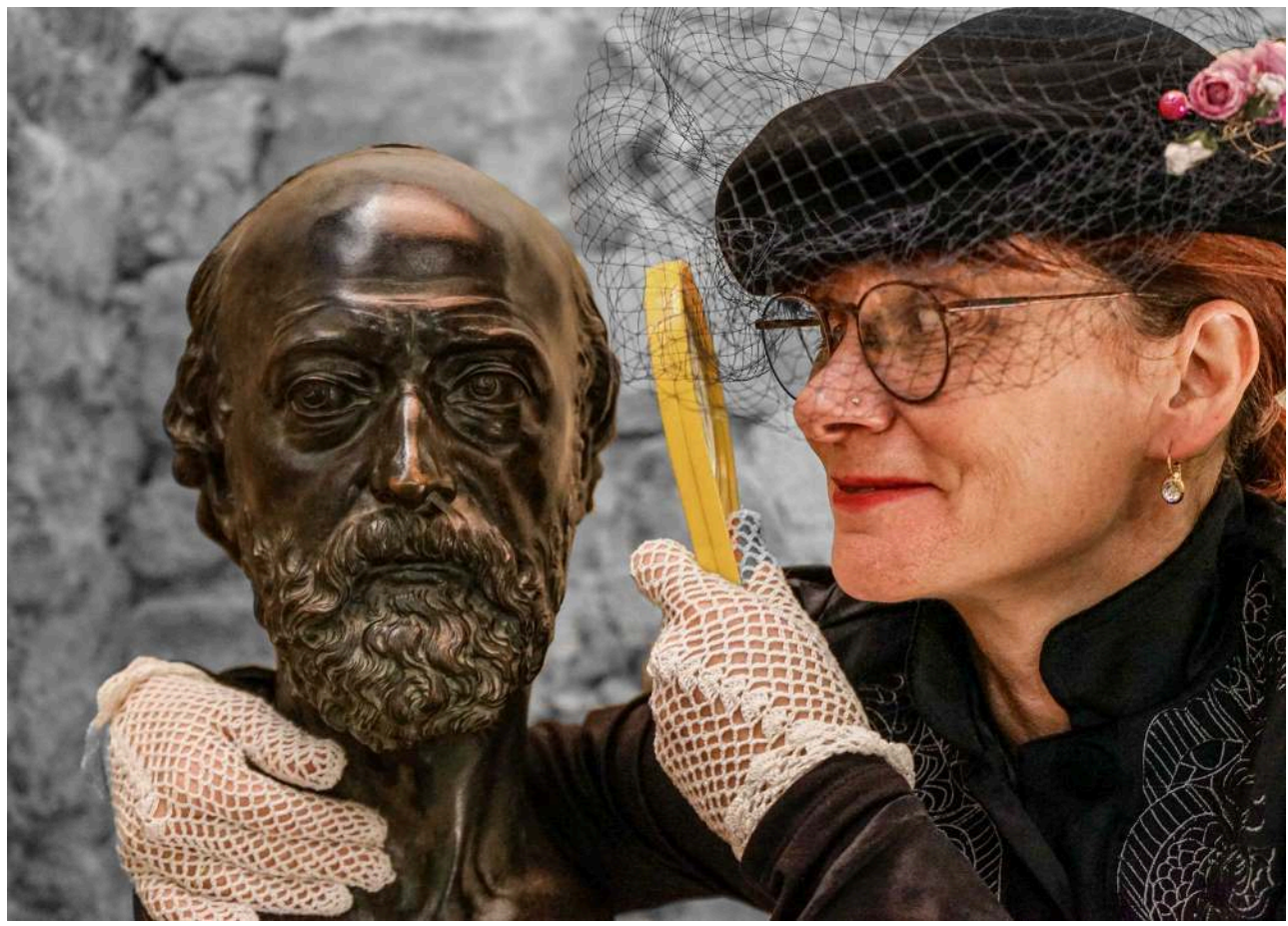

Violette Le-Truc, guide des remparts et du château de Carcassonne dans le cadre des visites famille (CMN, 2019). L'invention de ce personnage d'inspectrice des Monuments historiques, descendante imaginaire de Viollet-le-Duc, reflète la place centrale qu'occupent aujourd'hui les enfants dans la médiation - l'un des principaux thèmes du colloque de préfiguration du GIS «Patrimoines en partage » : «Enfants et patrimoines » (Lahic / Ethnopôle Garae, 2020).

(c) Catherine JeanJean (CMN)

Dans cet environnement singulier, le projet intellectuel du GIS s'est forgé et affermi à la faveur d'opérations scientifiques de préfiguration (colloques, séminaires, journées d'étude, projets des publications), qui ont été autant d'occasions d'établir de nouvelles coopérations scientifiques. Celles-ci ont fait place dans un deuxième temps à un recrutement plus direct des futurs partenaires, de manière à équiper le GIS de compétences diversifiées. Les partenariats d'ores et déjà esquissés visent en effet à créer les conditions d'une réflexion pluridisciplinaire (anthropologie, SIC, histoire, droit, etc.) et, en outre, à fournir un environnement propice au dialogue entre chercheurs en SHS (sciences humaines et sociales) et professionnels du patrimoine. Il s'agit à cet égard de prendre acte de tout le bénéfice déjà retiré des échanges noués par les uns et les autres autour des questions de patrimoine depuis plus d'une vingtaine d'années, sous l'égide de la Mission du patrimoine ethnologique (devenue Mission à l'ethnologie avant que ses compétences ne relèvent du DPRPS (département du Pilotage de la recherche et de la Politique scientifique) puis de la Diri (délégation à l'Inspection, à la Recherche et à l'Innovation), aussi bien dans le cadre du projet scientifique et culturel de l'Ethnopôle Garae, que dans le cadre des programmes du Lahic désormais déployés au sein de l'UMR Héritages nouvellement créée. De fait, les institutions partenaires du GIS relèvent à dessein de statuts divers: UMR (unités mixtes de recherches) ${ }^{1}$, EA (équipes d'accueil) ${ }^{2}$, labex (laboratoire d'excellence) ${ }^{3}$, établissements d'enseignement supérieur ${ }^{4}$ et institutions patrimoniales dépendant du ministère de la Culture $^{5}$, musées ${ }^{6}$, associations professionnelles ou culturelles ${ }^{7}$, collectivités territoriales ${ }^{8}$, etc. Cette manière d'hybridité trouvera à s'exprimer par ailleurs dans les 
instances de gouvernance ainsi que dans le portage du GIS. Double, celui-ci sera assumé d'une part par l'UMR Héritages (CNRS, université de Cergy Pontoise, ministère de la Culture), d'autre part par l'Ethnopôle Garae.

Figure 4

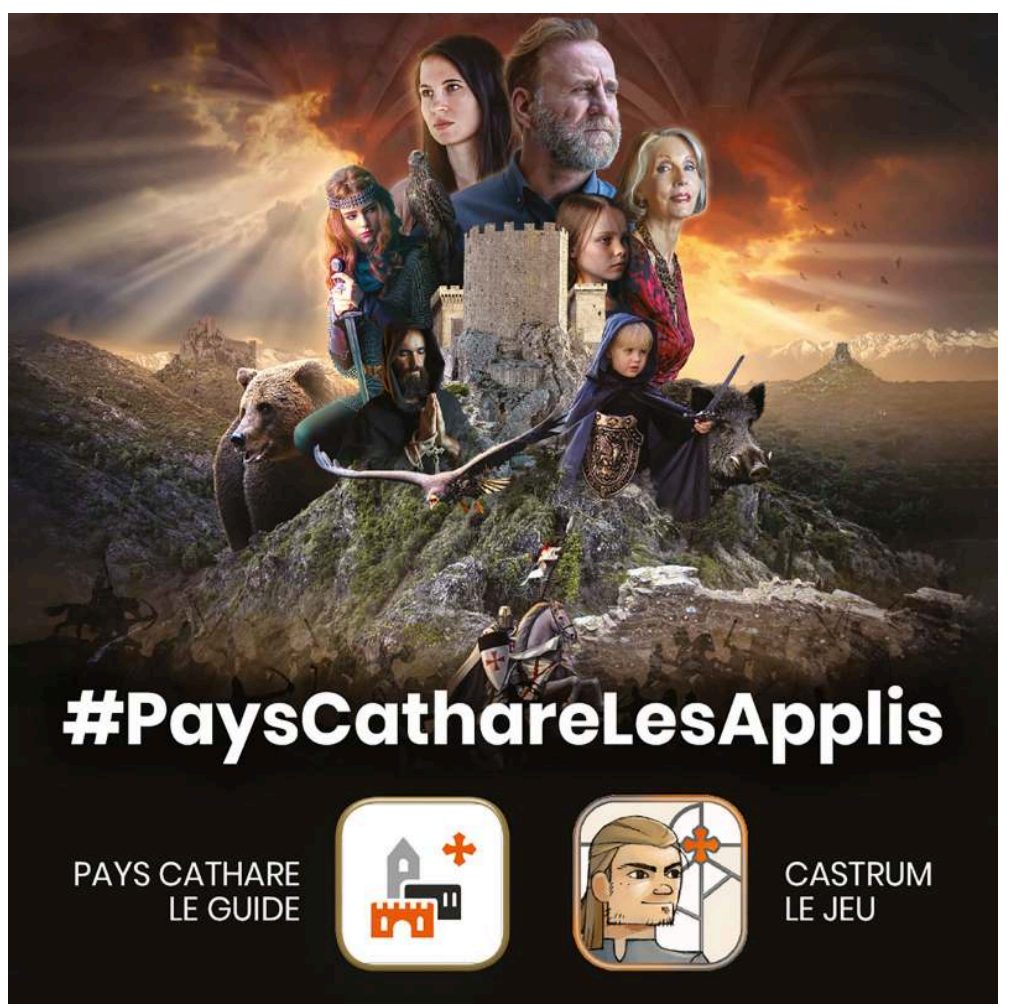

«Vous allez vivre ce que vous allez voir » en Pays cathare. Ce dispositif de médiation numérique est un exemple de médiation participative et expérientielle du patrimoine au cœur des préoccupations du GIS « Patrimoines en partage ». Applications mobiles proposées au téléchargement sur https:// www.payscathare.org/les-applis.

5 C'est sur ces bases, intellectuelles et organisationnelles, que sera signée en 2021 la convention sur laquelle le réseau reposera. Celle-ci intégrera un degré de souplesse suffisant pour permettre l'élargissement du GIS à d'autres partenaires afin que cette association soit en mesure de jouer pleinement le rôle qui lui est prioritairement dévolu: stimuler, dans le dialogue, les curiosités et interrogations de chacun, chercheurs et experts du patrimoine, de manière à toujours mieux cerner cette passion du passé, aujourd'hui comme jamais partagée aux quatre coins du monde. Cette formalité remplie et avec elle les conditions d'une réelle synergie, il ne s'agira plus que de faire fructifier les désirs de travailler ensemble à travers des projets communs. 


\section{BIBLIOGRAPHIE}

CAILLET Élisabeth, 1994, «L'ambiguïté de la médiation culturelle : entre savoir et présence », Publics et Musées, $n^{\circ}$ 6, p. 53-73. Disponible en ligne, https://www.persee.fr/doc/ pumus_1164-5385_1994_num_6_1_1046 [lien valide en janvier 2021].

FABRE Daniel, 2016, « L'ordinaire, le familier, l'intime... Le patrimoine loin du monument », in HOTTIN Christian \& VOISENAT Claudie (dir.), Le Tournant patrimonial. Mutations contemporaines des métiers du patrimoine, Paris, Éditions de la Maison des sciences de l'homme, coll. «Ethnologie de la France », p. 43-58.

\section{NOTES}

1. Outre Héritages: Patrimoine/s, Culture/s, Création/s, l'Idemec, le Centre Norbert-Élias et IrAsia (Institut de recherches asiatiques), TeMoS (Temps Mondes Sociétés),le Curapp (Centre universitaire de recherches sur l'action publique et le politique) et le Paloc (Patrimoines locaux, environnement et globalisation).

2. Outre le Canthel (Centre d'anthropologie culturelle), le Lerass (Laboratoire d'études et de recherches appliquées en sciences sociales), le Cresem (Centre de recherches sur les sociétés et environnements en Méditerranée), l'Item (Identités, Territoires, Expressions, Mobilités) et Habiter le monde.

3. Les passés dans le présent.

4. Université Toulouse - Jean-Jaurès, École polytechnique de Lausanne, INP (Institut national du patrimoine).

5. MuCEM (Musée des civilisations Europe et Méditerranée).

6. Latenium, parc et musée d'archéologie Hauterive-Neuchâtel.

7. SEF (Société d'ethnologie française), Ethnologues en herbe, Le Passe-muraille, Les ateliers Varan.

8. Archives départementales de l'Aude.

\section{AUTEUR}

\section{SYLVIE SAGNES}

Chargée de recherches CNRS (IIAC-Lahic), présidente de l'Ethnopôle GARAE

sylvie.sagnes@bbox.fr 